\title{
THE FRACTAL DIMENSION OF THE SPECTRUM OF THE FIBONACCI HAMILTONIAN
}

\author{
DAVID DAMANIK, MARK EMBREE, ANTON GORODETSKI, \\ AND SERGUEI TCHEREMCHANTSEV
}

\begin{abstract}
We study the spectrum of the Fibonacci Hamiltonian and prove upper and lower bounds for its fractal dimension in the large coupling regime. These bounds show that as $\lambda \rightarrow \infty, \operatorname{dim}\left(\sigma\left(H_{\lambda}\right)\right) \cdot \log \lambda$ converges to an explicit constant $(\approx 0.88137)$. We also discuss consequences of these results for the rate of propagation of a wavepacket that evolves according to Schrödinger dynamics generated by the Fibonacci Hamiltonian.
\end{abstract}

\section{INTRODUCTION}

The Fibonacci Hamiltonian is a discrete one-dimensional Schrödinger operator

$$
[H u](n)=u(n+1)+u(n-1)+V(n) u(n)
$$

in $\ell^{2}(\mathbb{Z})$. The potential $V: \mathbb{Z} \rightarrow \mathbb{R}$ is given by

$$
V(n)=\lambda \chi_{\left[1-\phi^{-1}, 1\right)}\left(n \phi^{-1}+\theta \bmod 1\right),
$$

where $\lambda>0$ is the coupling constant, $\phi$ is the golden mean,

$$
\phi=\frac{\sqrt{5}+1}{2}=1+\frac{1}{1+\frac{1}{1+\frac{1}{1+\cdots}}},
$$

and $\theta \in[0,1)$ is the phase.

This operator is important for both physical and mathematical reasons. On the one hand, it is the most popular quantum model of a one-dimensional quasicrystal, that is, a structure that shares many features with one displaying global order, but which in fact lacks global translation invariance. On the other hand, this operator has zero-measure Cantor spectrum and all spectral measures are purely singular continuous. These properties had been regarded as "exotic" in the context of general Schrödinger operators up until the 1980s, but for this operator family, they occur persistently for all parameter values. The Fibonacci Hamiltonian has been heavily studied since the early 1980s; see [7] for a recent review of the results obtained for it and related models.

Let us recall some specific results and references that will be important for what follows. It is known that the spectrum of the Fibonacci Hamiltonian is independent of $\theta$ (see, e.g., 4). This follows quickly from strong convergence once one realizes

Date: October 30, 2018.

D. D. was supported in part by NSF grant DMS-0653720. M. E. was supported by NSF grant DMS-CAREER-0449973. 
that for each pair $\theta, \tilde{\theta}$, there is a sequence $n_{k} \rightarrow \infty$ such that $\theta+n_{k} \phi^{-1}$ converges to $\tilde{\theta}$ in $\mathbb{R} / \mathbb{Z}$ "from the right." We denote this common spectrum by $\Sigma_{\lambda}$,

$$
\Sigma_{\lambda}=\sigma(H) \quad \text { for every } \theta \in[0,1) .
$$

It is natural to study the spectrum as a set. As we shall see, such a study is also motivated by the consequences one can draw for the long-time behavior of the solution of the time-dependent Schrödinger equation.

It has been shown by Sütö that the spectrum always has zero Lebesgue measure 35],

$$
\operatorname{Leb}\left(\Sigma_{\lambda}\right)=0 \quad \text { for every } \lambda>0 .
$$

This immediately implies the absence of absolutely continuous spectrum for all parameter values 1 It was later seen that one also has the absence of point spectrum for all parameter values; see Sütő [34, Hof-Knill-Simon [17, and Kaminaga 21] for partial results and Damanik-Lenz [9] for the full result. Thus, the Fibonacci model exhibits purely singular continuous spectrum that is very rigid in the sense that it is not affected by a change of the defining parameters.

The result on zero measure spectrum, (3), naturally leads one to ask about the dimension of this set. There are several popular ways to measure the fractal dimension of a nowhere dense subset of the real line.

Let us recall the definition of two of these dimensions. Suppose we are given a bounded and infinite set $S \subseteq \mathbb{R}$. A $\delta$-cover of $S$ is a countable union of real intervals, $\left\{I_{m}\right\}_{m \geq 1}$, such that each of these intervals has length bounded by $\delta>0$. For $\alpha \in[0,1]$, let

$$
h^{\alpha}(S)=\lim _{\delta \rightarrow 0} \inf _{\delta \text {-covers }} \sum_{m \geq 1}\left|I_{m}\right|^{\alpha} .
$$

It is clear that the limit exists in $[0, \infty]$. Moreover, if $h^{\alpha}(S)=0$ for some $\alpha$, then $h^{\alpha^{\prime}}(S)=0$ for every $\alpha^{\prime}>\alpha$. Similarly, if $h^{\alpha}(S)=\infty$ for some $\alpha$, then $h^{\alpha^{\prime}}(S)=\infty$ for every $\alpha^{\prime}<\alpha$. Thus, the following quantity is well-defined:

$$
\operatorname{dim}_{H}(S)=\inf \left\{\alpha: h^{\alpha}(S)<\infty\right\}=\sup \left\{\alpha: h^{\alpha}(S)=\infty\right\}
$$

The number $\operatorname{dim}_{H}(S) \in[0,1]$ is called the Hausdorff dimension of the set $S$.

A different way to measure the fractal dimension of $S$ is via the box counting dimension. The lower box counting dimension is defined as follows:

$$
\operatorname{dim}_{B}^{-}(S)=\liminf _{\varepsilon \rightarrow 0} \frac{\log N_{S}(\varepsilon)}{\log 1 / \varepsilon}
$$

where

$$
N_{S}(\varepsilon)=\#\{j \in \mathbb{Z}:[j \varepsilon,(j+1) \varepsilon) \cap S \neq \emptyset\} .
$$

The upper box counting dimension, $\operatorname{dim}_{B}^{+}(S)$, is defined similarly, with the liminf replaced by a limsup. When $\operatorname{dim}_{B}^{+}(S)$ and $\operatorname{dim}_{B}^{-}(S)$ are equal, we denote their common value by $\operatorname{dim}_{B}(S)$ and call this number the box counting dimension of $S$. These dimensions are related by the inequalities

$$
\operatorname{dim}_{H}(S) \leq \operatorname{dim}_{B}^{-}(S) \leq \operatorname{dim}_{B}^{+}(S) .
$$

In general, both inequalities may be strict; see, for example, [27, pp. 76-77].

\footnotetext{
${ }^{1}$ Historically, these two properties were established in the reverse order. The methods used by Kotani 24] in the proof of absence of absolutely continuous spectrum (for almost every $\theta$ ) were the key to proving zero measure spectrum.
} 
The main goal of this paper is to study the fractal dimension of the spectrum of the Fibonacci Hamiltonian. The following result shows that for sufficiently large coupling, the dimensions just introduced coincide.

Theorem 1. Suppose that $\lambda \geq 16$. Then the box counting dimension of $\Sigma_{\lambda}$ exists and obeys

$$
\operatorname{dim}_{B}\left(\Sigma_{\lambda}\right)=\operatorname{dim}_{H}\left(\Sigma_{\lambda}\right)
$$

While this theorem has not appeared in print explicitly before, it does follow quickly from a combination of known results. We present the relevant facts in the appendix.

Theorem 1 is useful because it will allow us to obtain precise asymptotics for the fractal dimension of $\Sigma_{\lambda}$ as $\lambda \rightarrow \infty$. The reason for this is the following. The box counting dimension is easier to bound from below, while the Hausdorff dimension is easier to bound from above. Consequently, we will prove a lower bound for the box counting dimension in Section 3 and an upper bound for the Hausdorff dimension in Section 4 .

It is known how to describe the spectrum of the Fibonacci Hamiltonian in terms of the spectra of canonical periodic approximants. We will recall this in Section 2. The general theory of periodic discrete one-dimensional Schrödinger operators shows that the spectrum of a such a periodic operator is always given by a finite union of compact intervals. Our crucial new insight is a way to describe the asymptotic distribution of bandwidths in these periodic spectra. In this description, the following function plays an important role. Define

$$
\begin{aligned}
f(x)=\frac{1}{x}[(2-3 x) \log 2+(1-x) \log (1-x) \\
-(2 x-1) \log (2 x-1)-(2-3 x) \log (2-3 x)]
\end{aligned}
$$

on the interval $\left(\frac{1}{2}, \frac{2}{3}\right)$. Setting $f\left(\frac{1}{2}\right)=\log 2$ and $f\left(\frac{2}{3}\right)=0$, it is not hard to see that $f$ extends to a continuous function on $\left[\frac{1}{2}, \frac{2}{3}\right]$, and with the aid of symbolic computation one can confirm that it takes its maximum at the unique point

$$
x^{*}=\frac{12-2 \sqrt{2}}{17}=0.5395042867796 \ldots,
$$

with

$$
f^{*}=f\left(x^{*}\right)=\log (1+\sqrt{2})=0.8813735870195 \ldots
$$

Write

$$
S_{u}(\lambda)=2 \lambda+22
$$

and

$$
S_{l}(\lambda)=\frac{1}{2}\left((\lambda-4)+\sqrt{(\lambda-4)^{2}-12}\right) .
$$

With these functions of (sufficiently large) $\lambda$ we can now state the bounds on the fractal dimension of $\Sigma_{\lambda}$ that we will prove in Sections 3 and 4 , respectively.

Theorem 2. (a) Suppose $\lambda>4$. Then

(b) Suppose $\lambda \geq 8$. Then

$$
\operatorname{dim}_{B}^{-}\left(\Sigma_{\lambda}\right) \geq \frac{f^{*}}{\log S_{u}(\lambda)} .
$$

$$
\operatorname{dim}_{H}\left(\Sigma_{\lambda}\right) \leq \frac{f^{*}}{\log S_{l}(\lambda)}
$$


Since both $S_{u}(\lambda)$ and $S_{l}(\lambda)$ behave asymptotically like $\log \lambda$, we obtain the following result as an immediate consequence. We write dim for either $\operatorname{dim}_{H}$ or $\operatorname{dim}_{B}$, which is justified by Theorem 1 .

Corollary 1. We have

$$
\lim _{\lambda \rightarrow \infty} \operatorname{dim}\left(\Sigma_{\lambda}\right) \cdot \log \lambda=f^{*} .
$$

In particular, we see that the constant $f^{*}$ is the best possible in both bounds in Theorem 2, Let us compare our results with previously known ones. To facilitate this, we introduce

$$
f^{\#}=\frac{f^{*}}{\log \phi}=\frac{\log (1+\sqrt{2})}{\log \phi}=1.8315709239073 \ldots,
$$

so that our results can be summarized as follows. We have for $\lambda \geq 16$,

$$
f^{\#} \frac{\log \phi}{\log S_{u}(\lambda)} \leq \operatorname{dim}_{B}\left(\Sigma_{\lambda}\right)=\operatorname{dim}_{H}\left(\Sigma_{\lambda}\right) \leq f^{\#} \frac{\log \phi}{\log S_{l}(\lambda)},
$$

and therefore the asymptotic behavior is

$$
\operatorname{dim}\left(\Sigma_{\lambda}\right) \sim f^{\#} \frac{\log \phi}{\log \lambda} .
$$

As we will see below, there are two competing scaling processes, one scaling with $\phi$ (the Fibonacci numbers) and one scaling with $\lambda$ (the inverse of the width of a band in the approximating periodic spectra). Thus, it is natural to write a bound in the form "constant times $\frac{\log \phi}{\log \lambda}$ " and then to optimize the constant.

Raymond 33 . proved an upper bound for $\operatorname{dim}_{H}\left(\Sigma_{\lambda}\right)$ that has a 2 in place of our $f^{\#}$ in (4). A simplified version of our approach (which we will comment on later in the paper) quickly gives a lower bound with $f^{\#}$ replaced in (44) by 1.5 and an upper bound with $f^{\#}$ replaced in (4) by 2 ; the latter being Raymond's result. These numbers appear naturally in this context and are associated with the support of a certain discrete probability distribution. A more detailed study of this distribution then led us to the discovery of $f^{\#}$, which describes the actual asymptotic behavior of the fractal dimension of the spectrum as we saw above.

Lower bounds for the dimension of the spectrum were initially obtained as a consequence of certain continuity properties of the spectral measures with respect to certain Hausdorff measures. Since the spectral measures are supported on the spectrum, one can obtain a lower bound for the Hausdorff dimension of the spectrum in this way. As mentioned above, this also bounds the box counting dimension from below by general principles. Spectral Hausdorff continuity results for the Fibonacci Hamiltonian were shown in [6, 8, 19, 22, The best lower bound that has been obtained in this way can be found in [22] and it reads

$$
\operatorname{dim}_{H}\left(\Sigma_{\lambda}\right) \geq \frac{2 \kappa}{\kappa+\zeta(\lambda)}
$$

where?

$$
\kappa=\frac{\log \left(\frac{\sqrt{17}}{4}\right)}{5 \log \phi} \approx 0.0126
$$

\footnotetext{
${ }^{2}$ Notice that there is a typo in 22 . They have $\kappa=\log \frac{\sqrt{17}}{20 \log \phi}$, a negative number!
} 
and

$$
\zeta(\lambda)=\frac{6 \log \sqrt{5}}{\log \phi}(\log \lambda+O(1)) .
$$

Thus, for $\lambda$ large, this gives a lower bound for $\operatorname{dim}_{H}\left(\Sigma_{\lambda}\right)$ as in (4), but with $f^{\#}$ replaced by

$$
\frac{2 \frac{\log \left(\frac{\sqrt{17}}{4}\right)}{5 \log \phi}}{6 \log \sqrt{5}} \approx 0.00188 .
$$

Liu and Wen 25] then extended the approach employed by Raymond. They study the case of general frequencies. Specialized to the Fibonacci case, their result shows that for $\lambda>20$,

$$
\frac{\log 2}{10 \log 2+3 \log (4(\lambda-8))} \leq \operatorname{dim}_{H}\left(\Sigma_{\lambda}\right) \leq \frac{\log 3}{\log \left(\frac{\lambda-8}{3}\right)} .
$$

Let us discuss this result in the large coupling limit. Since

$$
\frac{\log 3}{\log \phi} \approx 2.28301>2 \text {, }
$$

the upper bound does not improve Raymond's result. The lower bound has a constant coefficient

$$
\frac{\log 2}{3 \log \phi} \approx 0.48013
$$

in front of $\frac{\log \phi}{\log \lambda}$, a significant improvement over the result that can be extracted from [22]. Again, by our result, the optimal constant is $f^{\#} \approx 1.83157$.

Our interest in obtaining the optimal constant $f^{\#}$ does not only stem from natural curiosity. An interesting and mathematically challenging problem is to study the spreading of a wavepacket in a quantum system in the case where the initial state has a purely singular continuous spectral measure. This is the case for every initial state from $\ell^{2}(\mathbb{Z})$ for a system governed by the Fibonacci Hamiltonian. One is often especially interested in the spreading of a wavepacket that is initially localized on just one site.

That is, with $H$ as above, we consider $\psi(t)=e^{-i t H} \delta_{1}$ and study its spreading via the time-averaged outside probabilities

$$
P_{r}(N, T)=\sum_{n>N} \frac{2}{T} \int_{0}^{\infty} e^{-\frac{2 t}{T}}\left|\left\langle e^{-i t H} \delta_{1}, \delta_{n}\right\rangle\right|^{2} d t
$$

and

$$
P_{l}(N, T)=\sum_{n<-N} \frac{2}{T} \int_{0}^{\infty} e^{-\frac{2 t}{T}}\left|\left\langle e^{-i t H} \delta_{1}, \delta_{n}\right\rangle\right|^{2} d t .
$$

Let $P(N, T)=P_{l}(N, T)+P_{r}(N, T)$ and define

$$
S^{-}(\alpha)=-\liminf _{T \rightarrow \infty} \frac{\log P\left(T^{\alpha}-2, T\right)}{\log T}
$$

and

$$
S^{+}(\alpha)=-\limsup _{T \rightarrow \infty} \frac{\log P\left(T^{\alpha}-2, T\right)}{\log T} .
$$


For every $\alpha, 0 \leq S^{+}(\alpha) \leq S^{-}(\alpha) \leq \infty$. These numbers control the power decaying tails of the wavepacket. In particular, the following critical exponents are of interest:

$$
\alpha_{u}^{ \pm}=\sup \left\{\alpha \geq 0: S^{ \pm}(\alpha)<\infty\right\} .
$$

One can interpret $\alpha_{u}^{ \pm}$as the rates of propagation of the fastest (polynomially small) part of the wavepacket; compare [15. In particular, if $\alpha>\alpha_{u}^{+}$, then $P\left(T^{\alpha}, T\right)$ goes to 0 faster than any inverse power of $T$, and if $\alpha>\alpha_{u}^{-}$, then there is a sequence of times $T_{k} \rightarrow \infty$ such that $P\left(T_{k}^{\alpha}, T_{k}\right)$ goes to 0 faster than any inverse power of $T_{k}$.

In Section 5 we prove a result for general Schrödinger operators on $\ell^{2}(\mathbb{Z})$ that will imply the following consequence for the Fibonacci Hamiltonian.

Theorem 3. For every $\lambda>0$ and every $\theta \in[0,1)$, we have that

$$
\alpha_{u}^{ \pm} \geq \operatorname{dim}_{B}^{ \pm}\left(\Sigma_{\lambda}\right)
$$

Consequently, for $\lambda>4$ and every $\theta$, we have

$$
\alpha_{u}^{ \pm} \geq \frac{f^{*}}{\log S_{u}(\lambda)} .
$$

To discuss this result in the large coupling limit, let us be slightly inaccurat 3 and write

$$
\alpha_{u}^{ \pm} \geq f^{\#} \frac{\log \phi}{\log \lambda}
$$

There are two main previous approaches to quantum dynamical lower bounds for the Fibonacci model. The first is based on spectral continuity and the papers 6, 8, 19, 22 contain results obtained in this way. For $\theta=0$, the best bound is contained in 22 and it has (6) with $f^{\#} \approx 1.83157$ replaced by (5), that is, $\approx 0.00188$. For other values of $\theta$, the best bound can be found in 8 , and the constant in this bound is even smaller.

The other approach is based on complex energy methods and the Plancherel Theorem; see [11 (and also [12] for a way to combine the two approaches). For $\theta=0$, the paper [11] has (6) with $f^{\#}$ replaced by $1 / 6$. It is possible to treat general $\theta$ along the same lines using [10, but the dynamical lower bound has a somewhat smaller constant in the general case.

Thus, on the one hand, our result improves the constant from the previously best value $1 / 6$ to $f^{\#} \approx 1.83157$ and, on the other hand, this is the best one can do using the method put forth in this paper. We would like to mention that [13] contains the following upper bound for $\alpha_{u}^{+}$, which holds for $\lambda \geq 8$,

$$
\alpha_{u}^{+} \leq 2 \frac{\log \phi}{\log S_{l}(\lambda)} .
$$

While we know that the dimension of the spectrum indeed behave like $f^{\#} \frac{\log \phi}{\log \lambda}$ in the large coupling limit, we expect that the dynamical quantities $\alpha_{u}^{ \pm}$behaves like $2 \frac{\log \phi}{\log \lambda}$ in the large coupling limit. That is, we expect the following to hold,

$$
\lim _{\lambda \rightarrow \infty} \alpha_{u}^{ \pm} \frac{\log \lambda}{\log \phi}=2
$$

The reason for this is that the spreading of the fastest part of the wavepacket is determined by the "most continuous" part of the spectral measure and, in this case,

\footnotetext{
${ }^{3}$ The precise statement is that $\liminf _{\lambda \rightarrow \infty} \alpha_{u}^{ \pm} \frac{\log \lambda}{\log \phi} \geq f^{\#}$.
} 
by the region in the spectrum that is "the thickest." Since we will see that there is indeed a small region that is thickest in a natural sense, the factor 2 will then appear naturally. A forthcoming publication, 14, will deal with this issue using ideas and results from [13].

\section{The Band-Gap Structure of the Approximating Periodic Spectra}

In this section we describe the canonical coverings of $\Sigma_{\lambda}$ by (unions of) periodic spectra and the hierarchical structure of these sets. We use the combinatorics of this description to derive detailed results about the distribution of bandwidths in these spectra.

For $E \in \mathbb{R}$ and $\lambda>0$, we define a sequence of numbers $x_{k}=x_{k}(E, \lambda)$ as follows.

$$
x_{-1}=2, \quad x_{0}=E, \quad x_{1}=E-\lambda, \quad x_{k+1}=x_{k} x_{k-1}-x_{k-2} \quad \text { for } k \geq 1 .
$$

Using this recurrence and the initial values, one can quickly check (see Sütö [34]) that

$$
x_{k+1}^{2}+x_{k}^{2}+x_{k-1}^{2}-x_{k+1} x_{k} x_{k-1}=4+\lambda^{2} \text { for every } k \geq 0 .
$$

For fixed $\lambda>0$, define

$$
\sigma_{k}=\left\{E \in \mathbb{R}:\left|x_{k}(E, \lambda)\right| \leq 2\right\} .
$$

The set $\sigma_{k}$ is actually equal to the spectrum of the Schrödinger operator $H$ whose potential $V_{k}$ results from $V$ (with $\theta=0$ ) by replacing $\phi^{-1}$ with $F_{k-1} / F_{k}$ (cf. [34]). Here, $\left\{F_{k}\right\}_{k \geq 0}$ denotes the sequence of Fibonacci numbers, that is,

$$
F_{0}=1, F_{1}=1, F_{k+1}=F_{k}+F_{k-1} \text { for } k \geq 1 .
$$

Hence, $V_{k}$ is periodic and $\sigma_{k}$ consists of $F_{k}$ bands (closed intervals). Sütö also proved that

$$
\left(\sigma_{k-1} \cup \sigma_{k}\right) \supset\left(\sigma_{k} \cup \sigma_{k+1}\right)
$$

and

$$
\Sigma_{\lambda}=\bigcap_{k \geq 1}\left(\sigma_{k} \cup \sigma_{k+1}\right)=\left\{E:\left\{x_{k}\right\} \text { is a bounded sequence }\right\} .
$$

From now on, we assume

$$
\lambda>4,
$$

since we will make critical use of the fact that in this case, it follows from the invariant (8) that three consecutive $x_{k}$ 's cannot all be bounded in absolute value by 2 :

$$
\sigma_{k} \cap \sigma_{k+1} \cap \sigma_{k+2}=\emptyset .
$$

The identity (12) is the basis for work done by Raymond [33]; see also [13, 22, which describe the band structure on the various levels in an inductive way. Let us recall this result. Following [22], we call a band $I_{k} \subset \sigma_{k}$ a "type A band" if $I_{k} \subset \sigma_{k-1}$ (and hence $\left.I_{k} \cap\left(\sigma_{k+1} \cup \sigma_{k-2}\right)=\emptyset\right)$. We call a band $I_{k} \subset \sigma_{k}$ a "type B band" if $I_{k} \subset \sigma_{k-2}$ (and therefore $I_{k} \cap \sigma_{k-1}=\emptyset$ ). Then, we have the following result (Lemma 5.3 of [22], essentially Lemma 6.1 of [33]): 
Lemma 1. For every $\lambda>4$ and every $k \geq 1$,

(a) Every type $A$ band $I_{k} \subset \sigma_{k}$ contains exactly one type $B$ band $I_{k+2} \subset \sigma_{k+2}$, and no other bands from $\sigma_{k+1}, \sigma_{k+2}$.

(b) Every type $B$ band $I_{k} \subset \sigma_{k}$ contains exactly one type $A$ band $I_{k+1} \subset \sigma_{k+1}$ and two type $B$ bands from $\sigma_{k+2}$, positioned around $I_{k+1}$.

Lemma 2. For every band $I$ of $\sigma_{k}$, we have that $I \cap \Sigma_{\lambda} \neq \emptyset$.

Proof. Let $I$ be a band of $\sigma_{k}$. Choose a band $I^{(1)}$ in $\sigma_{k+1} \cup \sigma_{k+2}$ with $I \supset I^{(1)}$, as is possible by Lemma 1. Iterating this procedure, we obtain a nested sequence of intervals and hence a point $E \in I$ for which the corresponding trace map orbit is bounded. Thus, by (10), $E \in \Sigma_{\lambda}$.

We define

$$
\begin{aligned}
a_{k} & =\text { number of bands of type } \mathrm{A} \text { in } \sigma_{k}, \\
b_{k} & =\text { number of bands of type } \mathrm{B} \text { in } \sigma_{k} .
\end{aligned}
$$

By Raymond's work, it follows immediately that $a_{k}+b_{k}=F_{k}$ for every $k$. In fact, we have the following result.

Lemma 3. The constants $\left\{a_{k}\right\}$ and $\left\{b_{k}\right\}$ obey the relations

$$
a_{k}=b_{k-1}, \quad b_{k}=a_{k-2}+2 b_{k-2}
$$

with initial values

$$
a_{0}=1, \quad a_{1}=0, \quad b_{0}=0, \quad b_{1}=1 .
$$

Consequently, for $k \geq 2$,

$$
a_{k}=b_{k-1}=F_{k-2} .
$$

Proof. The recursions (13) hold by definition. The explicit expressions in (14) then follow quickly by induction.

We are interested in the size of the bands of a given type on a given level. To capture the distribution of these lengths, we define

$$
\begin{aligned}
& a_{k, m}=\text { number of bands } b \text { of type } \mathrm{A} \text { in } \sigma_{k} \text { with } \#\left\{0 \leq j<k: b \cap \sigma_{j} \neq \emptyset\right\}=m, \\
& b_{k, m}=\text { number of bands } b \text { of type } \mathrm{B} \text { in } \sigma_{k} \text { with } \#\left\{0 \leq j<k: b \cap \sigma_{j} \neq \emptyset\right\}=m .
\end{aligned}
$$

The motivation for this definition is the following: If we consider a given band and its location relative to the bands on the previous levels, each time the given band is contained in a band on a previous level, we essentially pick up a factor roughly of size $\lambda^{-1}$ (for $\lambda$ large). Thus, for example, there are $a_{k, m}$ bands of size $\approx \lambda^{-m}$ of type $A$ in $\sigma_{k}$. Since the combinatorics of the situation is $\lambda$-independent, we choose to separate the two aspects. This will allow us to get much more precise information in the regime of large $\lambda$, which is extremely hard to study numerically.

Lemma 4. We have

$$
a_{k, m}=b_{k-1, m-1}, \quad b_{k, m}=a_{k-2, m-1}+2 b_{k-2, m-1}
$$


with initial values

$$
\begin{aligned}
& a_{0, m}=0 \text { for } m>0 \quad \text { and } \quad a_{0,0}=1, \\
& a_{1, m}=0 \text { for } m \geq 0, \\
& b_{0, m}=0 \text { for } m \geq 0, \\
& b_{1, m}=0 \text { for } m>0 \quad \text { and } \quad b_{1,0}=1 .
\end{aligned}
$$

Consequently,

$$
a_{k, m}=b_{k-1, m-1}= \begin{cases}2^{2 k-3 m-1} \frac{m}{k-m}\left(\begin{array}{c}
k-m \\
2 m-k
\end{array}\right) & \text { when }\left\lceil\frac{k}{2}\right\rceil \leq m \leq\left\lfloor\frac{2 k}{3}\right\rfloor ; \\
0 & \text { otherwise. }\end{cases}
$$

Remark. The fact that $a_{k, m}$ is zero when $\left\lceil\frac{k}{2}\right\rceil \leq m \leq\left\lfloor\frac{2 k}{3}\right\rfloor$ fails is an immediate consequence of the properties (91), (10), and (12) established by Sütö. As a slight variation of the proof of Theorem 4 below shows, this fact alone is sufficient to give a quick proof of

$$
\operatorname{dim}_{B}^{ \pm}\left(\Sigma_{\lambda}\right) \geq 1.5 \frac{\log \phi}{\log S_{\mathrm{u}}(\lambda)}
$$

Our more detailed description of the numbers $a_{k, m}$ will then enable us to prove the stronger lower bound with $f^{\#}$ in place of 1.5 , which is optimal as discussed in the Introduction.

Proof. It is immediate from the definition and (13) that the recursions (15) hold. Recall that the Chebyshev polynomials of the first kind are defined by the recurrence relation

$$
\begin{aligned}
T_{0}(x) & =1, \\
T_{1}(x) & =x, \\
T_{m+1}(x) & =2 x T_{m}(x)-T_{m-1}(x) .
\end{aligned}
$$

Write $T_{m}(x)$ as

$$
T_{m}(x)=\sum_{r=0}^{\left\lfloor\frac{m}{2}\right\rfloor}(-1)^{r} c_{r, m} x^{m-2 r} .
$$

On the one hand, it is known (see, e.g., [1]) that for $m \geq 1$ and $0 \leq r \leq\left\lfloor\frac{m}{2}\right\rfloor$,

$$
c_{r, m}=2^{m-2 r-1} \frac{m}{m-r}\left(\begin{array}{c}
m-r \\
r
\end{array}\right) \text {. }
$$

On the other hand, the recursion generating the polynomials says that $c_{0,0}=1$, $c_{0,1}=1$, and

$$
\begin{aligned}
\sum_{r=0}^{\left\lfloor\frac{m+1}{2}\right\rfloor}(-1)^{r} c_{r, m+1} x^{m-2 r+1} & =2 x \sum_{r=0}^{\left\lfloor\frac{m}{2}\right\rfloor}(-1)^{r} c_{r, m} x^{m-2 r}-\sum_{r=0}^{\left\lfloor\frac{m-1}{2}\right\rfloor}(-1)^{r} c_{r, m-1} x^{m-2 r-1} \\
& =\sum_{r=0}^{\left\lfloor\frac{m}{2}\right\rfloor}(-1)^{r} 2 c_{r, m} x^{m-2 r+1}+\sum_{r=0}^{\left\lfloor\frac{m-1}{2}\right\rfloor}(-1)^{r+1} c_{r, m-1} x^{m-2 r-1} \\
& =\sum_{r=0}^{\left\lfloor\frac{m}{2}\right\rfloor}(-1)^{r} 2 c_{r, m} x^{m-2 r+1}+\sum_{r=1}^{\left\lfloor\frac{m+1}{2}\right\rfloor}(-1)^{r} c_{r-1, m-1} x^{m-2 r+1} .
\end{aligned}
$$


It follows that

$$
c_{r, m+1}=2 c_{r, m}+c_{r-1, m-1} .
$$

Denote $\tilde{a}_{k, m}=c_{2 m-k, m}$. Then, using (18), we find that

$$
\begin{aligned}
\tilde{a}_{k+1, m+1} & =c_{2(m+1)-(k+1), m+1} \\
& =c_{2 m-k+1, m+1} \\
& =2 c_{2 m-k+1, m}+c_{2 m-k, m-1} \\
& =2 \tilde{a}_{k-1, m}+\tilde{a}_{k-2, m-1} .
\end{aligned}
$$

Comparing this with the recursion

$$
a_{k+1, m+1}=2 a_{k-1, m}+a_{k-2, m-1}
$$

we established above, along with the initial values, we see that

$$
a_{k, m}=\tilde{a}_{k, m}=c_{2 m-k, m} .
$$

Combining (17) and (19), we obtain (16).

Recall that we introduced the function

$$
\begin{aligned}
f(x)=\frac{1}{x}[(2-3 x) \log 2+(1-x) \log (1-x) \\
\quad-(2 x-1) \log (2 x-1)-(2-3 x) \log (2-3 x)]
\end{aligned}
$$

on the interval $\left(\frac{1}{2}, \frac{2}{3}\right)$. We set $f\left(\frac{1}{2}\right)=\log 2$ and $f\left(\frac{2}{3}\right)=0$ and write

$$
x^{*}=\frac{12-2 \sqrt{2}}{17}=0.5395042867796 \ldots
$$

for the unique point in $\left(\frac{1}{2}, \frac{2}{3}\right)$ where $f$ takes its maximum value,

$$
f^{*}=f\left(x^{*}\right)=\log (1+\sqrt{2})=0.8813735870195 \ldots
$$

Proposition 1. For $\frac{k}{2} \leq m \leq \frac{2 k}{3}$, we have

$$
k^{-1 / 2} \exp \left(m f\left(\frac{m}{k}\right)\right) \lesssim a_{k, m} \lesssim k^{1 / 2} \exp \left(m f\left(\frac{m}{k}\right)\right) .
$$

Consequently,

$$
\lim _{k \rightarrow \infty} \max _{m} \frac{1}{m} \log a_{m, k}=f^{*}
$$

Proof. As we saw above, when $\frac{k}{2} \leq m \leq \frac{2 k}{3}$,

$$
a_{k, m}=2^{2 k-3 m-1} \frac{m}{k-m}\left(\begin{array}{c}
k-m \\
2 m-k
\end{array}\right) \text {. }
$$

Thus, we see that $a_{k, \frac{k}{2}}=2^{\frac{k}{2}-1}$ and $a_{k, \frac{2 k}{3}}=1$, so the estimate (20) holds when $m=\frac{k}{2}$ or $m=\frac{2 k}{3}$.

Let us now consider $\frac{k}{2}<m<\frac{2 k}{3}$, in which case

$$
\begin{aligned}
& \frac{k}{3}<k-m<\frac{k}{2} \\
& 1 \leq 2 m-k<\frac{k}{3} \\
& 1 \leq 2 k-3 m<\frac{k}{2} .
\end{aligned}
$$


Stirling's approximation gives

$$
n ! \asymp \sqrt{n}\left(\frac{n}{e}\right)^{n}
$$

for every $n \geq 1$, where we write $a \asymp b$ if $a \lesssim b$ and $a \gtrsim b$. Thus,

$$
a_{k, m} \asymp 2^{2 k-3 m} \frac{m}{k-m}\left(\frac{k-m}{(2 m-k)(2 k-3 m)}\right)^{1 / 2} \frac{\left(\frac{k-m}{e}\right)^{k-m}}{\left(\frac{2 m-k}{e}\right)^{2 m-k}\left(\frac{2 k-3 m}{e}\right)^{2 k-3 m}} .
$$

Due to the estimates (22)-(24), (20) will follow once we show that

$$
\tilde{a}_{k, m}:=2^{2 k-3 m} \frac{\left(\frac{k-m}{e}\right)^{k-m}}{\left(\frac{2 m-k}{e}\right)^{2 m-k}\left(\frac{2 k-3 m}{e}\right)^{2 k-3 m}}=\exp \left(m f\left(\frac{m}{k}\right)\right) .
$$

Writing $x=m / k$, we find that

$$
\begin{aligned}
\tilde{a}_{k, m} & =2^{2 k-3 m} \frac{(k-m)^{k-m}}{(2 m-k)^{2 m-k}(2 k-3 m)^{2 k-3 m}} \\
& =2^{2 k-3 k x} \frac{(k-k x)^{k-k x}}{(2 k x-k)^{2 k x-k}(2 k-3 k x)^{2 k-3 k x}} \\
& =2^{2 k-3 k x} \frac{(1-x)^{k(1-x)}}{(2 x-1)^{k(2 x-1)}(2-3 x)^{k(2-3 x)}} \\
& =\exp (k x f(x)) \\
& =\exp \left(m f\left(\frac{m}{k}\right)\right) .
\end{aligned}
$$

This finishes the proof of (20), which in turn immediately implies

$$
\limsup _{k \rightarrow \infty} \max _{m} \frac{1}{m} \log a_{m, k} \leq f^{*} .
$$

On the other hand, as $k$ gets large, we can choose $m$ so that $m / k$ gets arbitrarily close to $x^{*}$, so that by (20) again,

$$
\liminf _{k \rightarrow \infty} \max _{m} \frac{1}{m} \log a_{m, k} \geq f^{*} .
$$

Thus, we have established (21).

\section{A Lower Bound for the Box Counting Dimension of the Spectrum}

In this section we will prove a lower bound for the (lower) box counting dimension of the spectrum of the Fibonacci Hamiltonian. Recall that the lower box counting dimension of a bounded set $S \subset \mathbb{R}$ is defined as follows:

$$
\operatorname{dim}_{B}^{-}(S)=\liminf _{\varepsilon \rightarrow 0} \frac{\log N_{S}(\varepsilon)}{\log 1 / \varepsilon}
$$

where

$$
N_{S}(\varepsilon)=\#\{j \in \mathbb{Z}:[j \varepsilon,(j+1) \varepsilon) \cap S \neq \emptyset\} .
$$

The following was shown in [11]. 
Lemma 5. For every $\lambda>4$, there exists $S_{u}(\lambda)$ such that the following holds:

(a) Given any (type A) band $I_{k+1} \subset \sigma_{k+1}$ lying in the band $I_{k} \subset \sigma_{k}$, we have for every $E \in I_{k+1}$,

$$
\left|\frac{x_{k+1}^{\prime}(E)}{x_{k}^{\prime}(E)}\right| \leq S_{u}(\lambda)
$$

(b) Given any (type B) band $I_{k+2} \subset \sigma_{k+2}$ lying in the band $I_{k} \subset \sigma_{k}$, we have for every $E \in I_{k+2}$,

$$
\left|\frac{x_{k+2}^{\prime}(E)}{x_{k}^{\prime}(E)}\right| \leq S_{u}(\lambda)
$$

For example, one can choose $S_{u}(\lambda)=2 \lambda+22$.

We use the symbol $S_{u}(\lambda)$ instead of the explicit $2 \lambda+22$ to make the dependence of everything that follows on this quantity explicit.

Inductively, Lemma 5 gives an upper bound for $\left|x_{k}^{\prime}\right|$ on each band of $\sigma_{k}$ and this in turn will give a lower bound on the length of a band, which will be crucial in the proof of the following result.

Theorem 4. For all $\lambda>4$,

$$
\operatorname{dim}_{B}^{-}\left(\Sigma_{\lambda}\right) \geq \frac{f^{*}}{\log S_{u}(\lambda)}
$$

Proof. Let $m_{k}=\left\lfloor 3 k x^{*}\right\rfloor$. Since $x^{*}>0.4$, the sequence $\left\{m_{k}\right\}$ is strictly increasing and $\lim _{k \rightarrow \infty} \frac{m_{k}}{3 k}=x^{*}$. Write

$$
f_{k}=\frac{1}{m_{k}} \log a_{3 k, m_{k}} .
$$

We know from Proposition 1 that

$$
\lim _{k \rightarrow \infty} f_{k}=f^{*}
$$

and it is obvious that

$$
\lim _{k \rightarrow \infty} \frac{m_{k+1}}{m_{k}}=1
$$

For a given $k$, let us consider the type $\mathrm{A}$ bands in $\sigma_{3 k}$ that lie in $m_{k}$ bands on previous levels. By definition, there are $N_{k}:=a_{3 k, m_{k}}$ such bands. By Lemma 5 , each of them has length at least $\varepsilon_{k}:=4 S_{u}(\lambda)^{-m_{k}}$, since on each band of $\sigma_{3 k}$, the function $x_{3 k}$ is strictly monotone and runs from \pm 2 to $\mp 2$. Let $\left\{A_{3 k, j}\right\}_{j=1}^{N_{k}}$ be these bands, enumerated so that $A_{3 k, j}$ is to the left of $A_{3 k, j+1}$ for every $j$. Each band has non-empty intersection with $\Sigma_{\lambda}$ by Lemma 2, Thus, for every $j$, there exists $E_{3 k, j} \in A_{3 k, j} \cap \Sigma_{\lambda}$. Clearly, the energies $\left\{E_{3 k, j}\right\}$ are increasing in $j$. Consider $\left\{E_{3 k, j}\right\}$ with $j$ odd, that is, $j=2 s+1,0 \leq s \leq\left\lfloor N_{k} / 2\right\rfloor$. Since any two bands $A_{3 k, 2 s-1}, A_{3 k, 2 s+1}$ are separated by the band $A_{3 k, 2 s}$, which has length at least $\varepsilon_{k}$, we get $\left|x_{3 k, 2 s-1}-x_{3 k, 2 s+1}\right| \geq \varepsilon_{k}$ for every $s$. Thus, the $E_{3 k, 2 s+1}$ belong to different $\varepsilon$-boxes if $\varepsilon<\varepsilon_{k}$. We conclude that $N_{\Sigma_{\lambda}}(\varepsilon) \geq \frac{N_{k}}{2}$ for every $\varepsilon<\varepsilon_{k}$.

Given any $\varepsilon>0$, choose $k$ with $\varepsilon_{k+1} \leq \varepsilon<\varepsilon_{k}$. Then,

$$
\frac{\log N_{\Sigma_{\lambda}}(\varepsilon)}{\log 1 / \varepsilon} \geq \frac{\log \left(a_{3 k, m_{k}}\right)-\log 2}{\log 1 / \varepsilon_{k+1}} \geq \frac{f_{k}-\frac{1}{m_{k}} \log 2}{\frac{m_{k+1}}{m_{k}} \log S_{u}(\lambda)}
$$


Since, as $\varepsilon \rightarrow 0$, we have $k, m_{k} \rightarrow \infty$ and

$$
\lim _{k \rightarrow \infty} \frac{f_{k}}{\frac{m_{k+1}}{m_{k}} \log S_{u}(\lambda)}=\frac{f^{*}}{\log S_{u}(\lambda)},
$$

by (26) and (27), the result follows.

\section{An Upper Bound for the Hausdorff Dimension of the Spectrum}

In this section we prove an upper bound for the Hausdorff dimension of $\Sigma_{\lambda}$. We will use the canonical coverings $\sigma_{k} \cup \sigma_{k+1}$ of $\Sigma_{\lambda}$ and estimate the lengths of the intervals in these periodic spectra from above using our combinatorial result from Section 2 together with a scaling result that is analogous to Lemma 5 , but which gives bounds from the other side.

Namely, the following was shown by Killip, Kiselev, and Last [22, Lemma 5.5].

Lemma 6. For every $\lambda \geq 8$, there exists $S_{l}(\lambda)$ such that the following holds:

(a) Given any (type A) band $I_{k+1} \subset \sigma_{k+1}$ lying in the band $I_{k} \subset \sigma_{k}$, we have for every $E \in I_{k+1}$,

$$
\left|\frac{x_{k+1}^{\prime}(E)}{x_{k}^{\prime}(E)}\right| \geq S_{l}(\lambda)
$$

(b) Given any (type B) band $I_{k+2} \subset \sigma_{k+2}$ lying in the band $I_{k} \subset \sigma_{k}$, we have for every $E \in I_{k+2}$,

$$
\left|\frac{x_{k+2}^{\prime}(E)}{x_{k}^{\prime}(E)}\right| \geq S_{l}(\lambda)
$$

For example, one can choose

$$
S_{l}(\lambda)=\frac{1}{2}\left((\lambda-4)+\sqrt{(\lambda-4)^{2}-12}\right) .
$$

As before, we use the symbol $S_{l}(\lambda)$ instead of the explicit possible choice to make the dependence of everything that follows on this quantity explicit.

Inductively, Lemma 6 gives a lower bound for $\left|x_{k}^{\prime}\right|$ on each band of $\sigma_{k}$ and hence an upper bound for the length of a band.

Theorem 5. Suppose $\lambda \geq 8$. Then

$$
\operatorname{dim}_{H}\left(\Sigma_{\lambda}\right) \leq \frac{f^{*}}{\log S_{l}(\lambda)} .
$$

Remark. Raymond 33] proved an upper bound of the form

$$
\operatorname{dim}_{H}\left(\Sigma_{\lambda}\right) \leq \frac{2 \log \phi}{\log S_{l}(\lambda)} .
$$

As above, to get this weaker result, all one needs is information about the support of $a_{k, m}$, which in turn follows quickly from the properties (9), (10), and (12). Using our more detailed information about the values of $a_{k, m}$ on the support, we can improve the constant 2 to the value $f^{\#} \approx 1.83157$, which is optimal by our discussion in the introduction. Our proof is inspired by Raymond's proof, and the improvement stems from our more detailed analysis of the distributions of bandwidths in the approximating periodic spectra. 
Proof. As recalled above, the set $\sigma_{k} \cup \sigma_{k+1}$ is a finite union of compact real intervals that covers $\Sigma_{\lambda}$. There are $a_{k, m}+b_{k, m}$ bands in $\sigma_{k}$ that lie in exactly $m$ intervals on previous levels. We know that the length of each of these bands is bounded from above by $4 S_{l}(\lambda)^{-m}$.

Thus, it suffices to show that, given any

$$
s>\frac{f^{*}}{\log S_{l}(\lambda)},
$$

we have

$\lim _{k \rightarrow \infty} \sum_{m=\left\lceil\frac{k}{2}\right\rceil}^{\left\lfloor\frac{2 k}{3}\right\rfloor}\left(a_{k, m}+b_{k, m}\right)\left(4 S_{l}(\lambda)\right)^{-s m}+\sum_{m=\left\lceil\frac{k+1}{2}\right\rceil}^{\left\lfloor\frac{2(k+1)}{3}\right\rfloor}\left(a_{k+1, m}+b_{k+1, m}\right)\left(4 S_{l}(\lambda)\right)^{-s m}=0$.

For simplicity, we will only consider

$$
C_{k}=\sum_{m=\left\lceil\frac{k}{2}\right\rceil}^{\left\lfloor\frac{2 k}{3}\right\rfloor} a_{k, m} S_{l}(\lambda)^{-s m}
$$

the other terms can be dealt with in an analogous way.

By our upper bound for $a_{k, m}$ established earlier and the assumption (28), we have

$$
\begin{aligned}
C_{k} & \lesssim k^{1 / 2} \sum_{m=\left\lceil\frac{k}{2}\right\rceil}^{\left\lfloor\frac{2 k}{3}\right\rfloor} \exp \left(m\left(f\left(\frac{m}{k}\right)-s \log S_{l}(\lambda)\right)\right) \\
& \lesssim k^{3 / 2} \exp \left(\frac{k}{2}\left(f^{*}-s \log S_{l}(\lambda)\right)\right) .
\end{aligned}
$$

Using (28) again, we see that the right-hand side goes to zero as $k \rightarrow \infty$.

Theorem 4 and Theorem 5 together establish Theorem 2 from the Introduction.

\section{Transfer Matrices, Box Counting Dimension, and Wavepacket SPREADING}

In this section we will first consider general half-line Schrödinger operators and derive a number of consequences from polynomially bounded transfer matrices. We then turn to general Schrödinger operators on the line and use our half-line results to derive a lower bound for the exponent governing the spreading of the fastest part of the wavepacket in terms of the box counting dimension of the spectrum. These results are relevant in our context since for the Fibonacci potential, it is known that the transfer matrices are polynomially bounded for all parameter values and all energies in the spectrum.

Consider a discrete half-line Schrödinger operator on $\ell^{2}\left(\mathbb{Z}_{+}\right)$,

$$
\left[H_{+} u\right](n)=u(n+1)+u(n-1)+V(n) u(n),
$$

with Dirichlet boundary condition $u(0)=0$. Here, $V$ is a bounded real-valued function. $H_{+}$is a bounded self-adjoint operator in $\ell^{2}\left(\mathbb{Z}_{+}\right)$. The vector $\delta_{1}$ is cyclic and we denote its spectral measure by $\mu$.

For $z \in \mathbb{C}$ and $m, n \in \mathbb{Z}_{+}$, we denote by $T(n, m ; z)$ the transfer matrix associated with the difference equation

$$
u(n+1)+u(n-1)+V(n) u(n)=z u(n) .
$$


That is, $T(n, m ; z)$ is the unique unimodular $2 \times 2$ matrix for which we have

$$
\left(\begin{array}{c}
u(n+1) \\
u(n)
\end{array}\right)=T(n, m ; z)\left(\begin{array}{c}
u(m+1) \\
u(m)
\end{array}\right)
$$

for every solution of (30).

To study the spreading of a wavepacket with initial state $\delta_{1}$ under the dynamics generated by $H$, one usually considers for $p>0$,

$$
\left\langle|X|_{\delta_{1}}^{p}\right\rangle(T)=\frac{2}{T} \int_{0}^{\infty} e^{-2 t / T} \sum_{n \in \mathbb{Z}_{+}} n^{p}\left|\left\langle e^{-i t H_{+}} \delta_{1}, \delta_{n}\right\rangle\right|^{2} d t
$$

One is interested in the power-law growth of this quantity and therefore studies the lower transport exponent

$$
\beta_{\delta_{1}}^{-}(p)=\liminf _{T \rightarrow \infty} \frac{\log \left\langle|X|_{\delta_{1}}^{p}\right\rangle(T)}{p \log T}
$$

and the upper transport exponent

$$
\beta_{\delta_{1}}^{+}(p)=\limsup _{T \rightarrow \infty} \frac{\log \left\langle|X|_{\delta_{1}}^{p}\right\rangle(T)}{p \log T} .
$$

Both functions $\beta_{\delta_{1}}^{ \pm}(p)$ are nondecreasing in $p$ and hence the following limits exist:

$$
\alpha_{u}^{ \pm}=\lim _{p \rightarrow \infty} \beta_{\delta_{1}}^{ \pm}(p) .
$$

Alternatively (see [15, Theorem 4.1]), we also have $\alpha_{u}^{ \pm}=\sup \left\{\alpha \geq 0: S^{ \pm}(\alpha)<\infty\right\}$, where

$$
\begin{aligned}
& S^{-}(\alpha)=-\liminf _{T \rightarrow \infty} \frac{\log P_{+}\left(T^{\alpha}-2, T\right)}{\log T} \\
& S^{+}(\alpha)=-\limsup _{T \rightarrow \infty} \frac{\log P_{+}\left(T^{\alpha}-2, T\right)}{\log T}
\end{aligned}
$$

and

$$
P_{+}(N, T)=\sum_{n>N} \frac{2}{T} \int_{0}^{\infty} e^{-2 t / T}\left|\left\langle e^{-i t H_{+}} \delta_{1}, \delta_{n}\right\rangle\right|^{2} d t .
$$

The following result derives several consequences from the fact that the transfer matrices are polynomially bounded for energies from some subset of the real line. We denote by $\mu$ the spectral measure associated with the vector $\delta_{1}$.

Theorem 6. Consider a half-line Schrödinger operator $H_{+}$with bounded potential as above. Let $A \subset[-B, B], B>0$, and assume that there exist positive constants $C, \alpha$ such that for every $E \in A$ and every $N \geq 1$,

$$
\|T(n, m ; E)\| \leq C N^{\alpha} \text { for all } m, n \in[1, N] .
$$

(a) For every $\sigma>0$, there exists $\varepsilon_{0}(\sigma)>0$ such that

$$
\mu([E-\varepsilon, E+\varepsilon]) \geq D \varepsilon^{L}
$$

for $\varepsilon \in\left(0, \varepsilon_{0}\right)$ and $E \in A$. Here, $L=(1+\sigma)(1+3 \alpha)$ and $D$ depends only on $\sigma$.

(b) For any $p>0$, we have that

$$
\beta_{\delta_{1}}^{ \pm}(p) \geq\left(1+\frac{1}{p}\right) \operatorname{dim}_{B}^{ \pm}(A)-\frac{1+3 \alpha}{p} .
$$


In particular,

$$
\alpha_{u}^{ \pm} \geq \operatorname{dim}_{B}^{ \pm}(A)
$$

Proof. It was shown by Germinet, Kiselev and Tcheremchantsev [15, Proposition 2.1] that for any $M>0$,

$$
\mu([E-\varepsilon, E+\varepsilon]) \geq C_{1} \int_{E-\varepsilon / 2}^{E+\varepsilon / 2}\|T(N, 0 ; x)\|^{-2} d x-C_{2} \varepsilon^{M} .
$$

Here $\varepsilon \in(0,1), E \in[-B, B], N=\left\lfloor\varepsilon^{-1-\sigma}\right\rfloor, \sigma>0$, and the constants $C_{1}, C_{2}$ depend only on $B, M, \sigma$.

Let $E \in A$. To bound the integral in (35) from below, we need an upper bound for $\|T(N, 1 ; x)\|$ for $x$ close to $E$. This can be accomplished using a perturbative argument of Simon; compare [11, Lemma 2.1]. Namely, if

$$
D(N, E)=\sup _{1 \leq n, m \leq N}\|T(n, m ; E)\|,
$$

then for any $\delta \in \mathbb{C}$ and $1 \leq n \leq N$ we have

$$
\|T(n, 1 ; E+\delta)\| \leq D(N, E) \exp (D(N, E)|n| \delta) .
$$

By assumption, $D(N, E) \leq C N^{\alpha}$ for $E \in A$ and $N \geq 1$. Thus,

$$
\|T(N, 0 ; E+\delta)\| \leq C N^{\alpha} \exp \left(C N^{1+\alpha} \delta\right),
$$

and for any $\delta$ with $|\delta| \leq \gamma=1 / 2 N^{-1-\alpha}$, we get

$$
\|T(N, 1 ; E+\delta)\| \leq C^{\prime} N^{\alpha}
$$

with a uniform constant.

Since $N=\left\lfloor\varepsilon^{-1-\sigma}\right\rfloor, \varepsilon \in(0,1)$, we see that $\gamma<\varepsilon / 2$ for $\varepsilon$ small enough and therefore $[E-\gamma, E+\gamma] \subset[E-\varepsilon / 2, E+\varepsilon / 2]$. Using (35) and (36), we find

$$
\begin{aligned}
\mu([E-\varepsilon, E+\varepsilon]) & \geq C_{1} \int_{E-\gamma}^{E+\gamma}\|T(N, 0 ; x)\|^{-2} d x-C_{2} \varepsilon^{M} \\
& \geq C_{3} \gamma N^{-2 \alpha}-C_{2} \varepsilon^{M} \\
& =C_{4} N^{-1-3 \alpha}-C_{2} \varepsilon^{M} \\
& =C_{4} \varepsilon^{(1+\sigma)(1+3 \alpha)}-C_{2} \varepsilon^{M}
\end{aligned}
$$

with appropriate constants. Taking $M=(1+\sigma)(1+3 \alpha)+1$, we obtain (32).

In order to prove the dynamical lower bound (33), we will apply [37, Corollary 4.1]; see also [2. In our notation, this result reads

$$
\beta_{\delta_{1}}^{ \pm}(p) \geq D_{\mu}^{ \pm}(q), q \in\left(\frac{1}{1+p}, 1\right),
$$

where $D_{\mu}(q), 0<q<1$ are the generalized fractal dimensions of the spectral measure; compare [2, 3]. One of the equivalent ways of defining these dimensions is the following (cf. [37, Theorem 4.3]):

$$
D_{\mu}^{+}(q)=\limsup _{\varepsilon \rightarrow 0} \frac{\log S_{\mu}(q, \varepsilon)}{(q-1) \log \varepsilon},
$$

and similarly for $D_{\mu}^{-}(q)$ with lim sup replaced by liminf, where

$$
S_{\mu}(q, \varepsilon)=\sum_{j \in \mathbb{Z}}\left(\mu([j \varepsilon,(j+1) \varepsilon))^{q} .\right.
$$


To bound $S_{\mu}(q, \varepsilon)$ from below, we can follow the proof of [37, Theorem 4.5]. Let $I_{j}=[j \varepsilon,(j+1) \varepsilon)$. Denote by $J_{A}$ the set of $j \in \mathbb{Z}$ for which $I_{j} \cap A \neq \emptyset$. Thus, for every $j \in J_{A}$, there exists $E_{j} \in\left[j \varepsilon,(j+1) \varepsilon\right.$ ) such that $E_{j} \in A$. The bound (32) implies for $\varepsilon$ small enough,

$$
\mu\left(I_{j-1}\right)+\mu\left(I_{j}\right)+\mu\left(I_{j+1}\right) \geq \mu\left(\left[E_{j}-\varepsilon, E_{j}+\varepsilon\right]\right) \geq D \varepsilon^{L},
$$

for every $j \in J_{A}$, where $L=(1+\sigma)(1+3 \alpha)$. Thus,

$$
B \equiv \sum_{j \in \mathbb{Z}}\left(\mu\left(I_{j-1}\right)+\mu\left(I_{j}\right)+\mu\left(I_{j+1}\right)\right)^{q} \geq D^{q} \varepsilon^{q L} \sum_{j \in J_{A}} 1 .
$$

On the other hand, since $(a+b)^{q} \leq a^{q}+b^{q}, a, b>0, q \in(0,1)$, one has $B \leq$ $3 S_{\mu}(q, \varepsilon)$. Therefore,

$$
S_{\mu}(q, \varepsilon) \geq C(q) \varepsilon^{q L} \sum_{j \in J_{A}} 1 \equiv C(q) \varepsilon^{q L} N(\varepsilon)
$$

with

$$
N(\varepsilon)=\#\left\{j \in \mathbb{Z}: I_{j} \cap A \neq \emptyset\right\} .
$$

It follows from (38)-(39) that

$$
D_{\mu}^{ \pm}(q) \geq \frac{\operatorname{dim}_{B}^{ \pm}(A)-q L}{1-q}, \quad q \in(0,1) .
$$

The bounds (37) and (40) imply, for any $q \in\left(\frac{1}{1+p}, 1\right)$,

$$
\beta_{\delta_{1}}^{ \pm}(p) \geq \frac{\operatorname{dim}_{B}^{ \pm}(A)-q L}{1-q} .
$$

Since this holds for $L=(1+\sigma)(1+3 \alpha)$ with any $\sigma>0$, letting $\sigma \rightarrow 0$ and $q \rightarrow \frac{1}{1+p}$, we obtain (33).

Since

$$
\alpha_{u}^{ \pm}=\lim _{p \rightarrow+\infty} \beta_{\delta_{1}}^{ \pm}(p)
$$

the estimate (34) follows.

Remark. One can improve the bounds (32), (33) if one has a nontrivial (i.e., better than ballistic) upper bound on $\alpha_{u}^{+}$. In this case [38, (35) holds with $N=\left\lfloor\varepsilon^{-\rho}\right\rfloor$, where $\rho=\alpha_{u}^{+}+\sigma, \sigma>0$. Thus, (32) holds with a smaller value of $L$.

Let us now turn to the whole-line case and prove a result analogous to (34). Consider the operator $H$ acting on $\ell^{2}(\mathbb{Z})$ as

$$
[H u](n)=u(n+1)+u(n-1)+V(n) u(n)
$$

with bounded $V: \mathbb{Z} \rightarrow \mathbb{R}$, and choose $K$ so that $\sigma(H) \subset[-K+1, K-1]$. The transfer matrices $T(n, m ; z)$ are defined in an analogous way.

For $N \geq 1$, recall that the time-averaged right outside probabilities are given by

$$
P_{r}(N, T)=\frac{2}{T} \int_{0}^{\infty} e^{-\frac{2 t}{T}} \sum_{n>N}\left|\left\langle e^{-i t H} \delta_{1}, \delta_{n}\right\rangle\right|^{2} d t .
$$

It was proved in [13] that

$$
C T^{-3} I_{r}(N, T) \leq P_{r}(N, T) \leq C e^{-c N}+C T^{3} I_{r}(N, T),
$$


where

$$
I_{r}(N, T)=\int_{-K}^{K}\left\|T\left(N, 1 ; E+\frac{i}{T}\right)\right\|^{-2} d E .
$$

Although not stated explicitly, this result from [13] can be extended easily to the half-line case (29). Thus, for the time-averaged outside probabilities $P_{+}(N, T)$, we have

$$
C T^{-3} I_{+}(N, T) \leq P_{+}(N, T) \leq C e^{-c N}+C T^{3} I_{+}(N, T)
$$

where

$$
I_{+}(N, T)=\int_{-K}^{K}\left\|T\left(N, 1 ; E+\frac{i}{T}\right)\right\|^{-2} d E .
$$

Corollary 2. Suppose that $H$ is a discrete Schrödinger operator on the line with bounded potential. Assume $A \subset[-B, B] \subset \mathbb{R}$ is such that for $E \in A$ and $N \geq 1$,

$$
\|T(n, m ; E)\| \leq C N^{\alpha} \text { for all } m, n \in[1, N]
$$

with uniform constants $C, \alpha$. Then, for the initial state $\delta_{1}$, we have that

$$
\alpha_{u}^{ \pm} \geq \operatorname{dim}_{B}^{ \pm}(A)
$$

Proof. Let $H_{+}$be the half-line operator of the form (29) with potential $V_{+}$given by $V_{+}(n)=V(n)$ for $n \geq 1$. The transfer matrices are the same for both operators since they are defined locally, and thus we have $I_{r}(N, T)=I_{+}(N, T)$. The condition (31) holds for the operator $H_{+}$and Theorem 6 therefore yields

$$
\alpha_{u,+}^{ \pm} \geq \operatorname{dim}_{B}^{ \pm}(A)
$$

for the corresponding dynamics.

Let us assume that $\operatorname{dim}_{B}^{-}(A)>0$ (if $\operatorname{dim}_{B}^{-}(A)=0$, the result is trivially true) and show that $\alpha_{u}^{-} \geq \operatorname{dim}_{B}^{-}(A)$. Take any $\alpha$ with $0<\alpha<\operatorname{dim}_{B}^{-}(A) \leq \alpha_{u,+}^{-}$. Due to the definition of $\alpha_{u,+}^{ \pm}$, we have that

$$
P_{+}\left(T^{\alpha}-2, T\right) \geq T^{-M}
$$

with some finite $M>0$ for $T$ sufficiently large. Since $I_{r}(N, T)=I_{+}(N, T)$, it follows from (41) and (42) that

$$
P_{r}(N, T) \geq C T^{-3} I_{+}(N, T) \geq C T^{-6}\left(P_{+}(N, T)-C e^{-c N}\right) .
$$

It follows from (44) that for $T$ sufficiently large,

$$
P_{r}\left(T^{\alpha}-2, T\right) \geq C T^{-M-6} \text {. }
$$

Consequently, for the full time-averaged outside probabilities, we find

$$
\begin{aligned}
P\left(T^{\alpha}-2, T\right) & =\frac{2}{T} \int_{0}^{\infty} e^{-\frac{2 t}{T}} \sum_{|n|>T^{\alpha}}\left|\left\langle e^{-i t H} \delta_{1}, \delta_{n}\right\rangle\right|^{2} d t \\
& \geq C T^{-M-6} .
\end{aligned}
$$

It now follows directly from the definition of $\alpha_{u}^{-}$that $\alpha_{u}^{-} \geq \alpha$. Since this holds for every $\alpha<\operatorname{dim}_{B}^{-}(A)$, the result follows. For $\alpha_{u}^{+}$, the proof is the completely analogous. (The bound (44) then holds for some sequence of times.) 
Remarks. (a) One can prove a similar result under the condition

$$
\|T(n, m ; E)\| \leq C N^{\alpha} \text { for all } m, n \in[-N,-1],
$$

where $E \in A, N \geq 1$.

(b) This establishes Theorem 3 as stated in the Introduction since it has been shown that in the Fibonacci case, the transfer matrices are polynomially bounded for all energies in the spectrum with uniform constants $C, \alpha$. See Iochum-Testard [18] for the case $\theta=0$ and Damanik-Lenz [10] for the case of general $\theta$.

Appendix A. The Hyperbolicity of the Trace Map at Large Coupling And Some of its Consequences

A.1. Description of the Trace Map. The main tool that we are using here is the trace map. It was originally introduced in 20, 23; see also 34 for proofs of the results described below. Let us recall that the numbers $x_{k}=x_{k}(E, \lambda)$ introduced in Section 2 satisfy the recursion relation

$$
x_{k+1}=x_{k} x_{k-1}-x_{k-2},
$$

with initial conditions $x_{-1}=2, x_{0}=E, x_{1}=E-\lambda$, and the invariance relation

$$
x_{k+1}^{2}+x_{k}^{2}+x_{k-1}^{2}-x_{k+1} x_{k} x_{k-1}=4+\lambda^{2}
$$

for every $k \in \mathbb{Z}_{+}$. Because of (45), it is natural to consider the so-called trace map,

$$
T: \mathbb{R}^{3} \rightarrow \mathbb{R}^{3}, T(x, y, z)=(x y-z, x, y) .
$$

The sequence $\left\{x_{1}, x_{2}, x_{3}, \ldots\right\}$ can be considered as the sequence of first coordinates of points in the trace map orbit having initial condition $\left(x_{1}, x_{0}, x_{-1}\right)$.

By (46), the following function is invariant under the action of $T$ :

$$
I=x^{2}+y^{2}+z^{2}-x y z-4 .
$$

In other words, $T$ preserves the family of cubic surfaces

$$
\mathbb{S}_{I}=\left\{(x, y, z) \in \mathbb{R}^{3}: x^{2}+y^{2}+z^{2}-x y z-4=I\right\} .
$$

The surface $\mathbb{S}_{0}$ is called the Cayley cubic. Denote by $l_{\lambda}$ the line

$$
l_{\lambda}=\{(E-\lambda, E, 2): E \in \mathbb{R}\} .
$$

It is easy to check that $l_{\lambda} \subset \mathbb{S}_{\lambda^{2}}$.

The following result, proved in 34, characterizes the spectrum of the Fibonacci Hamiltonian in terms of trace map dynamics and therefore establishes an important and fruitful connection between spectral and dynamical issues in this context.

Theorem 7. The energy $E$ belongs to the spectrum $\Sigma_{\lambda}$ of the Fibonacci Hamiltonian if and only if the positive semiorbit of the point $(E-\lambda, E, 2)$ under iterates of the trace map $T$ is bounded. 
A.2. Hyperbolicity of the Trace Map for Large $\lambda$. Denote by $f_{\lambda}$ the restriction of the trace map to the invariant surface $\mathbb{S}_{\lambda^{2}}$. That is, $f_{\lambda}: \mathbb{S}_{\lambda^{2}} \rightarrow \mathbb{S}_{\lambda^{2}}$ and $f_{\lambda}=\left.T\right|_{\mathbb{S}^{2}}$. Denote by $\Omega_{\lambda}$ the set of points in $\mathbb{S}_{\lambda^{2}}$ whose full orbits under $f_{\lambda}$ are bounded.

Let us recall that an invariant set $\Lambda$ of a diffeomorphism $f: M \rightarrow M$ is locally maximal if there exists a neighborhood $U(\Lambda)$ such that

$$
\Lambda=\bigcap_{n \in \mathbb{Z}} f^{n}(U)
$$

An invariant closed set $\Lambda$ of a diffeomorphism $f: M \rightarrow M$ is hyperbolic if there exists a splitting of the tangent space $T_{x} M=E_{x}^{s} \oplus E_{x}^{u}$ at every point $x \in \Lambda$ such that it is invariant under $D f$, and $D f$ exponentially contracts vectors from the stable subspaces $\left\{E_{x}^{s}\right\}$ and exponentially expands vectors from the unstable subspaces $\left\{E_{x}^{u}\right\}$.

See [16] for a detailed survey of hyperbolic dynamics and an extensive list of references.

Casadgli proved the following result [5].

Theorem 8. For every $\lambda \geq 16$, the set $\Omega_{\lambda}$ is a locally maximal invariant hyperbolic set of $f_{\lambda}: \mathbb{S}_{\lambda^{2}} \rightarrow \mathbb{S}_{\lambda^{2}}$.

A.3. Some Properties of Locally Maximal Hyperbolic Invariant Sets of Surface Diffeomorphisms. Consider a locally maximal invariant transitive hyperbolic set $\Lambda \subset M, \operatorname{dim} M=2$, of a diffeomorphism $f \in \operatorname{Diff}^{r}(M), r \geq 1$. We have $\Lambda=\cap_{n \in \mathbb{Z}} f^{n}(U(\Lambda))$ for some neighborhood $U(\Lambda)$. Assume also that $\operatorname{dim} E^{u}=\operatorname{dim} E^{s}=1$.

Let us gather several results in this general context that we will eventually specialize to the case where $\Lambda$ is given by $\Omega_{\lambda}$ for $f_{\lambda}: \mathbb{S}_{\lambda^{2}} \rightarrow \mathbb{S}_{\lambda^{2}}, \lambda \geq 16$.

I. Stability. There is a neighborhood $\mathcal{U} \subset \operatorname{Diff}^{1}(M)$ of the map $f$ such that for every $g \in \mathcal{U}$, the set $\Lambda_{g}=\cap_{n \in \mathbb{Z}} g(U(\Lambda))$ is a locally maximal invariant hyperbolic set of $g$. Moreover, there is a homeomorphism $h: \Lambda \rightarrow \Lambda_{g}$ that conjugates $\left.f\right|_{\Lambda}$ and $\left.g\right|_{\Lambda_{g}}$, that is, the following diagram commutes:

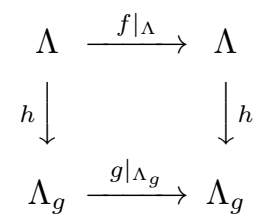

II. Invariant Manifolds. For $x \in \Lambda$ and small $\varepsilon>0$, consider the local stable and unstable sets

$$
\begin{aligned}
& W_{\varepsilon}^{s}(x)=\left\{w \in M: d\left(f^{n}(x), f^{n}(w)\right) \leq \varepsilon \text { for all } n \geq 0\right\}, \\
& W_{\varepsilon}^{u}(x)=\left\{w \in M: d\left(f^{n}(x), f^{n}(w)\right) \leq \varepsilon \text { for all } n \leq 0\right\} .
\end{aligned}
$$

If $\varepsilon>0$ is small enough, then these are embedded $C^{r}$-disks with $T_{x} W_{\varepsilon}^{s}(x)=E_{x}^{s}$ and $T_{x} W_{\varepsilon}^{u}(x)=E_{x}^{u}$. Define the (global) stable and unstable sets as

$$
W^{s}(x)=\cup_{n \in \mathbb{Z}_{+}} f^{-n}\left(W_{\varepsilon}^{s}(x)\right), \quad W^{u}(x)=\cup_{n \in \mathbb{Z}_{+}} f^{n}\left(W_{\varepsilon}^{u}(x)\right) .
$$

Define also

$$
W^{s}(\Lambda)=\cup_{x \in \Lambda} W^{s}(x) \quad \text { and } \quad W^{u}(\Lambda)=\cup_{x \in \Lambda} W^{u}(x) .
$$


III. Invariant Foliations. A stable foliation for $\Lambda$ is a foliation $\mathcal{F}^{s}$ of a neighborhood of $\Lambda$ such that

(a) for each $x \in \Lambda, \mathcal{F}(x)$, the leaf containing $x$, is tangent to $E_{x}^{s}$;

(b) for each $x \in \Lambda$, sufficiently near $\Lambda, f\left(\mathcal{F}^{s}(x)\right) \subset \mathcal{F}^{s}(f(x))$.

An unstable foliation $\mathcal{F}^{u}$ can be defined in a similar way.

For a locally maximal hyperbolic set $\Lambda \subset M$ of a $C^{1}$-diffeomorphism $f: M \rightarrow M$, $\operatorname{dim} M=2$, stable and unstable $C^{0}$ foliations with $C^{1}$-leaves can be constructed [26]. In the case of $C^{2}$-diffeomorphisms, $C^{1}$ invariant foliations exist (see [30, Theorem 8 in Appendix 1).

IV. Local Hausdorff Dimension and Box Counting Dimension. Consider, for $x \in \Lambda$ and small $\varepsilon>0$, the set $W_{\varepsilon}^{u}(x) \cap \Lambda$. The Hausdorff dimension of this set does not depend on $x \in \Lambda$ and $\varepsilon>0$, and coincides with its box counting dimension (see [28, 36]):

$$
\operatorname{dim}_{H} W_{\varepsilon}^{u}(x) \cap \Lambda=\operatorname{dim}_{B} W_{\varepsilon}^{u}(x) \cap \Lambda .
$$

In a similar way,

$$
\operatorname{dim}_{H} W_{\varepsilon}^{s}(x) \cap \Lambda=\operatorname{dim}_{B} W_{\varepsilon}^{s}(x) \cap \Lambda .
$$

Denote $h^{s}=\operatorname{dim}_{H} W_{\varepsilon}^{s}(x) \cap \Lambda$ and $h^{u}=\operatorname{dim}_{H} W_{\varepsilon}^{u}(x) \cap \Lambda$. We will call $h^{s}$ and $h^{u}$ the local stable and unstable Hausdorff dimensions of $\Lambda$, respectively.

V. Global Hausdorff Dimension. Moreover, the Hausdorff dimension of $\Lambda$ is equal to its box counting dimension and

$$
\operatorname{dim}_{H} \Lambda=\operatorname{dim}_{B} \Lambda=h^{s}+h^{u}
$$

see [28, 31.

VI. Continuity of the Hausdorff Dimension. The local Hausdorff dimensions $h^{s}(\Lambda)$ and $h^{u}(\Lambda)$ depend continuously on $f: M \rightarrow M$ in the $C^{1}$-topology; see 28, 31. Therefore, $\operatorname{dim}_{H} \Lambda_{f}=\operatorname{dim}_{B} \Lambda_{f}=h^{s}\left(\Lambda_{f}\right)+h^{u}\left(\Lambda_{f}\right)$ also depends continuously on $f$ in the $C^{1}$-topology. Moreover, for $r \geq 2$ and $C^{r}$-diffeomorphisms $f: M \rightarrow M$, the Hausdorff dimension of a hyperbolic set $\Lambda_{f}$ is a $C^{r-1}$ function of $f$; see [26].

Remark. For hyperbolic sets in dimension greater than two, most of these properties do not hold in general; see [32] for more details.

A.4. Implications for the Trace Map and the Spectrum. Due to Theorem 8 the properties I-VI can all be applied to the hyperbolic set $\Omega_{\lambda}$ of the trace map $f_{\lambda}: \mathbb{S}_{\lambda^{2}} \rightarrow \mathbb{S}_{\lambda^{2}}$ for every $\lambda \geq 16$. One can extract the following statement from the material in [5, Section 2].

Lemma 7. For $\lambda \geq 16$ and every $x \in \Omega_{\lambda}$, the stable manifold $W^{s}(x)$ intersects the line $l_{\lambda}$ transversally.

The existence of a $C^{1}$-foliation $\mathcal{F}^{s}$ allows us to locally consider the set $W^{s}\left(\Omega_{\lambda}\right) \cap l_{\lambda}$ as a $C^{1}$-image of a set $W_{\varepsilon}^{u}(x) \cap \Omega_{\lambda}$. Therefore, we obtain the following consequences for the spectrum of the Fibonacci Hamiltonian.

Theorem 9. For $\lambda \geq 16$, the following statements hold:

(a) The spectrum $\Sigma_{\lambda}$ depends continuously on $\lambda$ in the Hausdorff metric.

(b) We have $\operatorname{dim}_{H}\left(\Sigma_{\lambda}\right)=\operatorname{dim}_{B}\left(\Sigma_{\lambda}\right)$.

(c) For every small $\varepsilon>0$ and every $E \in \Sigma_{\lambda}$, we have

$$
\operatorname{dim}_{H}\left((E-\varepsilon, E+\varepsilon) \cap \Sigma_{\lambda}\right)=\operatorname{dim}_{H}\left(\Sigma_{\lambda}\right)
$$


and

$$
\operatorname{dim}_{B}\left((E-\varepsilon, E+\varepsilon) \cap \Sigma_{\lambda}\right)=\operatorname{dim}_{B}\left(\Sigma_{\lambda}\right) .
$$

(d) The Hausdorff dimension $\operatorname{dim}_{H}\left(\Sigma_{\lambda}\right)$ is a $C^{\infty}$-function of $\lambda$.

In particular, part (b) establishes Theorem 1 as formulated in the Introduction.

\section{REFERENCES}

[1] M. Abramowitz and I. Stegun, Handbook of Mathematical Functions with Formulas, Graphs, and Mathematical Tables, Dover, New York, 1965

[2] J.-M. Barbaroux, F. Germinet, and S. Tcheremchantsev, Fractal dimensions and the phenomenon of intermittency in quantum dynamics, Duke Math. J. 110 (2001), 161-193

[3] J.-M. Barbaroux, F. Germinet, and S. Tcheremchantsev, Generalized fractal dimensions: equivalences and basic properties, J. Math. Pures Appl. 80 (2001), 977-1012

[4] J. Bellissard, B. Iochum, E. Scoppola, and D. Testard, Spectral properties of one-dimensional quasicrystals, Commun. Math. Phys. 125 (1989), 527-543

[5] M. Casdagli, Symbolic dynamics for the renormalization map of a quasiperiodic Schrödinger equation, Commun. Math. Phys. 107 (1986), 295-318

[6] D. Damanik, $\alpha$-continuity properties of one-dimensional quasicrystals, Commun. Math. Phys. 192 (1998), 169-182

[7] D. Damanik, Strictly ergodic subshifts and associated operators, in Spectral Theory and Mathematical Physics: A Festschrift in Honor of Barry Simon's 60th Birthday, 505-538, Proceedings of Symposia in Pure Mathematics 74, American Mathematical Society, Providence, 2006

[8] D. Damanik, R. Killip, and D. Lenz, Uniform spectral properties of one-dimensional quasicrystals. III. $\alpha$-continuity, Commun. Math. Phys. 212 (2000), 191-204

[9] D. Damanik and D. Lenz, Uniform spectral properties of one-dimensional quasicrystals. I. Absence of eigenvalues, Commun. Math. Phys. 207 (1999), 687-696

[10] D. Damanik and D. Lenz, Uniform spectral properties of one-dimensional quasicrystals. II. The Lyapunov exponent, Lett. Math. Phys. 50 (1999), 245-257

[11] D. Damanik and S. Tcheremchantsev, Power-Law bounds on transfer matrices and quantum dynamics in one dimension, Commun. Math. Phys. 236 (2003), 513-534

[12] D. Damanik and S. Tcheremchantsev, Scaling estimates for solutions and dynamical lower bounds on wavepacket spreading, J. Anal. Math. 97 (2005), 103-131

[13] D. Damanik and S. Tcheremchantsev, Upper bounds in quantum dynamics, J. Amer. Math. Soc. 20 (2007), 799-827

[14] D. Damanik and S. Tcheremchantsev, in preparation

[15] F. Germinet, A. Kiselev, and S. Tcheremchantsev, Transfer matrices and transport for Schrödinger operators, Ann. Inst. Fourier (Grenoble), 54 (2004), 787-830

[16] B. Hasselblatt, Hyperbolic dynamical systems, in Handbook of Dynamical Systems, Vol. 1A, 239-319, North-Holland, Amsterdam, 2002

[17] A. Hof, O. Knill, and B. Simon, Singular continuous spectrum for palindromic Schrd̈inger operators, Commun. Math. Phys. 174 (1995), 149-159

[18] B. Iochum and D. Testard, Power law growth for the resistance in the Fibonacci model, $J$. Stat. Phys. 65 (1991), 715-723

[19] S. Jitomirskaya and Y. Last, Power law subordinacy and singular spectra. II. Line operators Commun. Math. Phys. 211 (2000), 643-658

[20] L. Kadanoff, Analysis of cycles for a volume preserving map, unpublished

[21] M. Kaminaga, Absence of point spectrum for a class of discrete Schrödinger operators with quasiperiodic potential, Forum Math. 8 (1996), 63-69

[22] R. Killip, A. Kiselev, and Y. Last, Dynamical upper bounds on wavepacket spreading, Amer. J. Math. 125 (2003), 1165-1198

[23] M. Kohmoto, L. P. Kadanoff, and C. Tang, Localization problem in one dimension: Mapping and escape, Phys. Rev. Lett. 50 (1983), 1870-1872

[24] S. Kotani, Jacobi matrices with random potentials taking finitely many values, Rev. Math. Phys. 1 (1989), 129-133

[25] Q.-H. Liu and Z.-Y. Wen, Hausdorff dimension of spectrum of one-dimensional Schrödinger operator with Sturmian potentials, Potential Anal. 20 (2004), 33-59 
[26] R. Mañé, The Hausdorff dimension of horseshoes of diffeomorphisms of surfaces, Bol. Soc. Brasil. Mat. (N.S.) 20 (1990), 1-24

[27] P. Mattila, Geometry of Sets and Measures in Euclidean Spaces. Fractals and Rectifiability, Cambridge University Press, Cambridge, 1995

[28] H. McCluskey and A. Manning, Hausdorff dimension for horseshoes, Ergodic Theory Dynam. Systems, 3 (1983), 251-261; Erratum, Ergodic Theory Dynam. Systems, 5 (1985), 319

[29] S. Ostlund, R. Pandit, D. Rand, H. J. Schellnhuber, and E. D. Siggia, One-dimensional Schrödinger equation with an almost periodic potential, Phys. Rev. Lett. 50 (1983), 18731877

[30] J. Palis and F. Takens, Hyperbolicity and Sensitive Chaotic Dynamics at Homoclinic Bifurcations. Fractal Dimensions and Infinitely Many Attractors, Cambridge University Press, Cambridge, 1993

[31] J. Palis and M. Viana, On the continuity of the Hausdorff dimension and limit capacity for horseshoes, in Dynamical Systems, Lecture Notes in Mathematics 1331, 150-160, Springer, Berlin, 1988

[32] Ya. Pesin, Dimension Theory in Dynamical Systems, University of Chicago Press, Chicago, 1997

[33] L. Raymond, A constructive gap labelling for the discrete Schrödinger operator on a quasiperiodic chain, preprint (1997)

[34] A. Sütö, The spectrum of a quasiperiodic Schrödinger operator, Commun. Math. Phys. 111 (1987), 409-415

[35] A. Sütö, Singular continuous spectrum on a Cantor set of zero Lebesgue measure for the Fibonacci Hamiltonian, J. Statist. Phys. 56 (1989), 525-531

[36] F. Takens, Limit capacity and Hausdorff dimension of dynamically defined Cantor sets, in Dynamical Systems, Lecture Notes in Mathematics 1331, 196-212, Springer, Berlin, 1988

[37] S. Tcheremchantsev, Mixed lower bounds for quantum transport, J. Funct. Anal. 197 (2003), $247-282$

[38] S. Tcheremchantsev, in preparation

Department of Mathematics, Rice University, Houston, TX 77005, USA

E-mail address: damanik@rice.edu

Computational and Applied Mathematics, Rice University, Houston, TX 77005, USA

E-mail address: embree@rice.edu

Mathematics 253-37, California Institute of Technology, Pasadena, CA 91125, USA

E-mail address: asgor@caltech.edu

Université d'Orléans, Laboratoire MAPMO, CNRS-UMR 6628, B.P. 6759, F-45067

Orléans Cedex, France

E-mail address: serguei.tcheremchantsev@univ-orleans.fr 